\title{
Evaluation of the Healing Process of Autogenous Tooth Bone Graft Material Nine Months after Sinus Bone Graft: Micromorphometric and Histological Evaluation
}

\author{
Young-Kyun Kim, Sang-Ho Jun ${ }^{1}$, In-Woong $\mathrm{Um}^{2}$, Sooyeon Kim ${ }^{3}$ \\ Department of Oral and Maxillofacial Surgery, Section of Dentistry, Seoul National University Bundang Hospital, \\ ${ }^{1}$ Department of Oral and Maxillofacial Surgery, Section of Dentistry, Korea University Anam Hospital, \\ ${ }^{2}$ R\&D Institute Korea Tooth Bank, ${ }^{3}$ KOREA INTERNATIONAL (GR.11)
}

\begin{abstract}
Micromorphometric and histological examinations were conducted with a collected tissue specimen nine months after sinus bone graft using autogenous tooth bone graft material (AutoBT). As a result of micro-computed tomographic analysis, the total bone volume (graft material+new bone) was $76.45 \%$, and the proportion of new bone was $45.4 \%$. The bone mineral density and the average Hounsfield Unit of new bone were 0.26 and 1,164.69, respectively. The histological examination showed that AutoBT particles were united well with new bone. AutoBT was considered to have excellent bone healing ability after sinus graft and bone density that can resist repneumatization.
\end{abstract}

Key words: Autogenous tooth, Bone, Sinus

\section{Introduction}

Any material whose resorption speed is not too high and whose bone healing process approximates that of autogenous bone graft may be useful in maxillary sinus bone grafting. Likewise, a more excellent clinical outcome might be expected when this material is used in combination with other bone substitutes with slow resorption properties[1-3]. Autogenous tooth bone graft material (AutoBT; Korea Tooth Bank, Seoul, Korea) was proven to exhibit bone healing ability through osteoinduction and osteoconduction, demonstrating a histological healing process similar to that of free bone grafting being resorbed over
3 6 months. Accordingly, AutoBT is regarded as a potential substitute when autogenous bone is needed for sinus bone graft, and it may have a useful effect on increasing the volume of bone graft materials and minimizing repneumatization[4]. AutoBT is a brand name of autogenous tooth bone graft material.

The authors sought to investigate the healing process through micromorphometric and histological examination with a collected tissue specimen nine months after sinus bone graft using powder-type autogenous tooth bone graft material. This histologic study and the consent forms were approved by SNUBH IRB (E-1110-067-003).

RECEIVED May 5, 2013, REVISED May 22, 2013, ACCEPTED September 21, 2013

Correspondence to Young-Kyun Kim

Department of Oral and Maxillofacial Surgery, Section of Dentistry, Seoul National University Bundang Hospital

82 Gumi-ro 173 beon-gil, Bundang-gu, Seongnam 463-707, Korea

Tel: 82-31-787-7541, Fax: 82-31-787-4068, E-mail: kyk0505@snubh.org

(c) This is an open access article distributed under the terms of the Creative Commons Attribution Non-Commercial License (http://creativecommons. org/licenses/ by-nc/3.0) which permits unrestricted non-commercial use, distribution, and reproduction in any medium, provided the original work is properly cited. 


\section{Case Report}

\section{Case report}

A 45-year-old male patient visited Seoul National University Bundang Hospital for implant treatment on the edentulous first and second molar areas in the left maxilla. The radiological examination revealed a mucous retention cyst in the left maxillary sinus; however, there were no clinical symptoms. The residual bone height to sinus floor

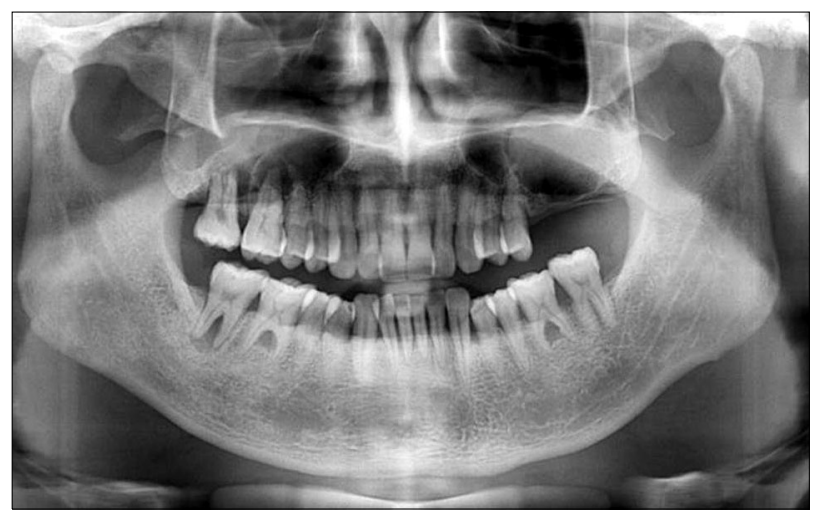

Fig. 1. Initial panoramic radiograph. The residual bone height of the left maxillary posterior area is definitely insufficient, showing a progressive periodontal disease. was definitely insufficient, therefore, sinus bone graft and delayed implant placement were planned (Fig. 1, 2); \#15, 16, and 17 teeth were extracted before surgery, and powder-type autogenous tooth bone graft material was made and stored. Sinus membrane elevation was performed through the lateral window approach, and a sinus bone graft was performed using AutoBT powder. After surgery, the areas healed normally without occurrence of any specific complication. Mucoperiosteal flap elevation was performed for implant placement after nine months. Two tissue specimens were collected from the areas where the

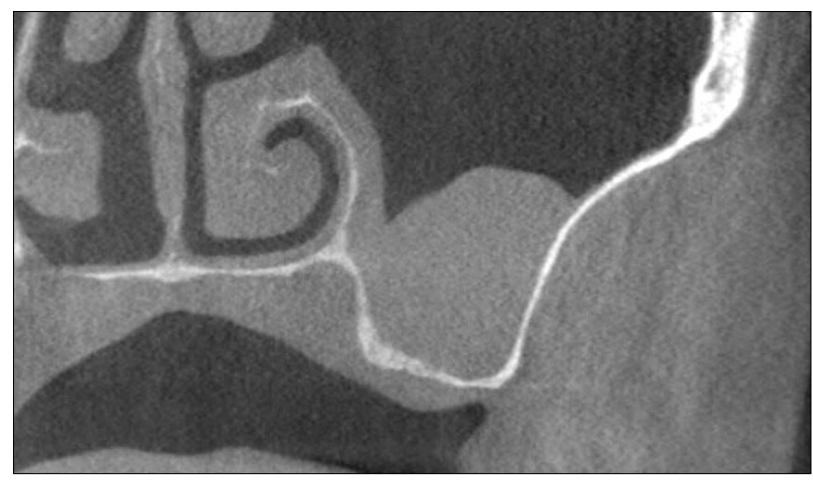

Fig. 2. Initial computed tomography. Mucosal thickening and dome-shaped radiopacity of the left maxillary sinus were observed.
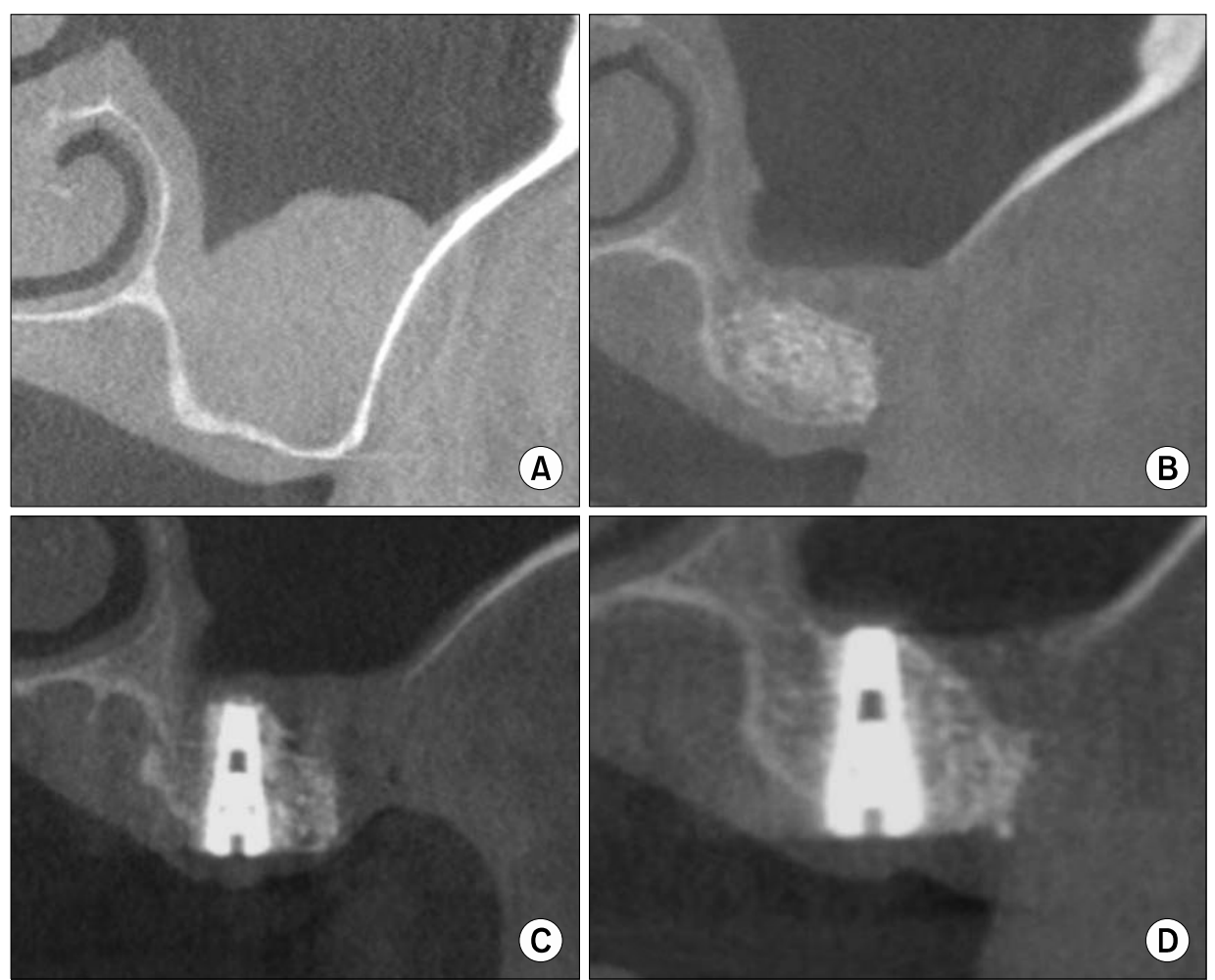

Fig. 3. Computed tomography (CT) examination of sinus bone graft. (A) CT view before surgery. Dome-shaped radiopacity and sinus mucosal thickening were observed. (B) CT view nine months after sinus bone graft. (C) CT view after implant placement. (D) CT view seven months after implant placement (16 months after sinus bone graft). 
implant was to be placed (\#26, 27) using a trephine bur with an external diameter of $3.0 \mathrm{~mm}$. Two implants (Perio Implant; DIO, Busan, Korea) were then placed, and wound closure was performed. After a healing period of approximately seven months, the implants were exposed, and the healing abutment was connected through the second surgery (Fig. 3).

\section{Micromorphometric and histologic examination}

The tissues contained in the trephine bur were stored in formalin, and a block embedded with methacrylate-based chemical curing resin was made through the washing and dehydration process for tissue sample fabrication and micro CT scanning. Trephine bur and external resin were carefully removed using a diamond bur without causing damage to the tissues (Fig. 4). The cylindrical specimen obtained was scanned using micro computed tomography (MicroCT) (Skyscan 1172; Bruker-Microct, Kontich, Belgium). Three-dimensional image reconstruction was performed for the reconstructed data, two-dimensional and three-dimensional image analyses were performed using the CTAn analysis program (Fig. 5). Hounsfield unit (HU) and bone mineral density (BMD) were calibrated using phantom in order to eliminate errors of each specimen. Regional objective image (ROI) was formed from the taken core sample, except that the autogenous bone and total HU and BMD were obtained from the binary image. HU more than 350 was regarded as new bone. HU of grafted material was obtained

A

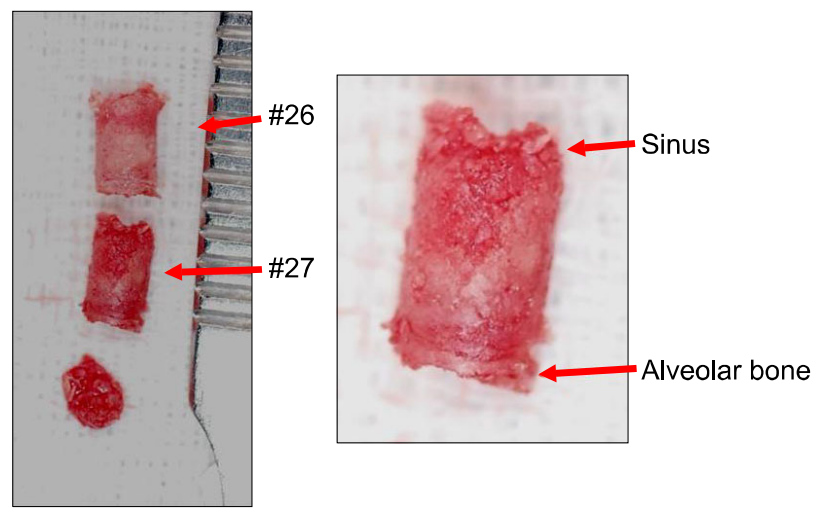

Fig. 4. Biopsy specimen. (A) Two tissue specimens were collected from areas \#26, 27. (B) Alveolar bone of approximately $1 \mathrm{~mm}$ thickness was observed, and osseous tissue showing a dense appearance was created upward and mixed with autogenous tooth bone particles. through analysis of binary image peak and HU more than 2,100 was eliminated from the data. This analytical method was based on Tajima et al.'s method[5].

The specimen that had undergone MicroCT scanning was secondarily embedded using Technovit4000VLC, and a tissue sample of 30 40 $\mu \mathrm{m}$ thickness was prepared by grinding the resin block. For the histological analysis, the healing status was evaluated through Masson trichrome staining, toluidine blue staining, and H\&E staining.

\section{Results}

As a result of MicroCT analysis, the total bone volume (graft material+new bone) of the bone tissue sample in the maxillary sinus was $76.45 \%$, and the proportion of new bone was 45.4\%. Total core BMD was 0.35 and BMD of new bone was 0.26. Average $\mathrm{HU}$ of the total core was $1,555.07$ and average HU of new bone was 1,164.69. Average trabecular thickness of new bone was 0.08282 $\mathrm{mm}$. On the other hand, based on the histological examination, formation of new bone and rising toward the maxillary sinus from the left alveolar bone was observed, and new bone and AutoBT particles were mixed in the middle area. Likewise, the examination showed a mixture of AutoBT and fibrous connective tissue in the roof area of the maxillary sinus (Fig. 6, 7).

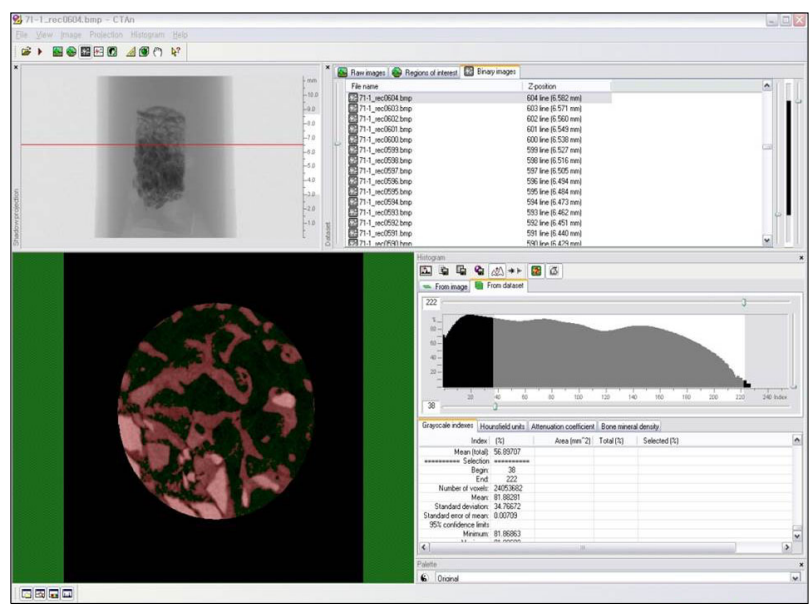

Fig. 5. The cylindrical specimen obtained was scanned using micro computed tomography (Skyscan 1172, Bruker-Microct, Kontich, Belgium). Three-dimensional image reconstruction was performed for the data, with two-dimensional and three-dimensional image analyses performed for the reconstructed data using the CTAn analysis program. 

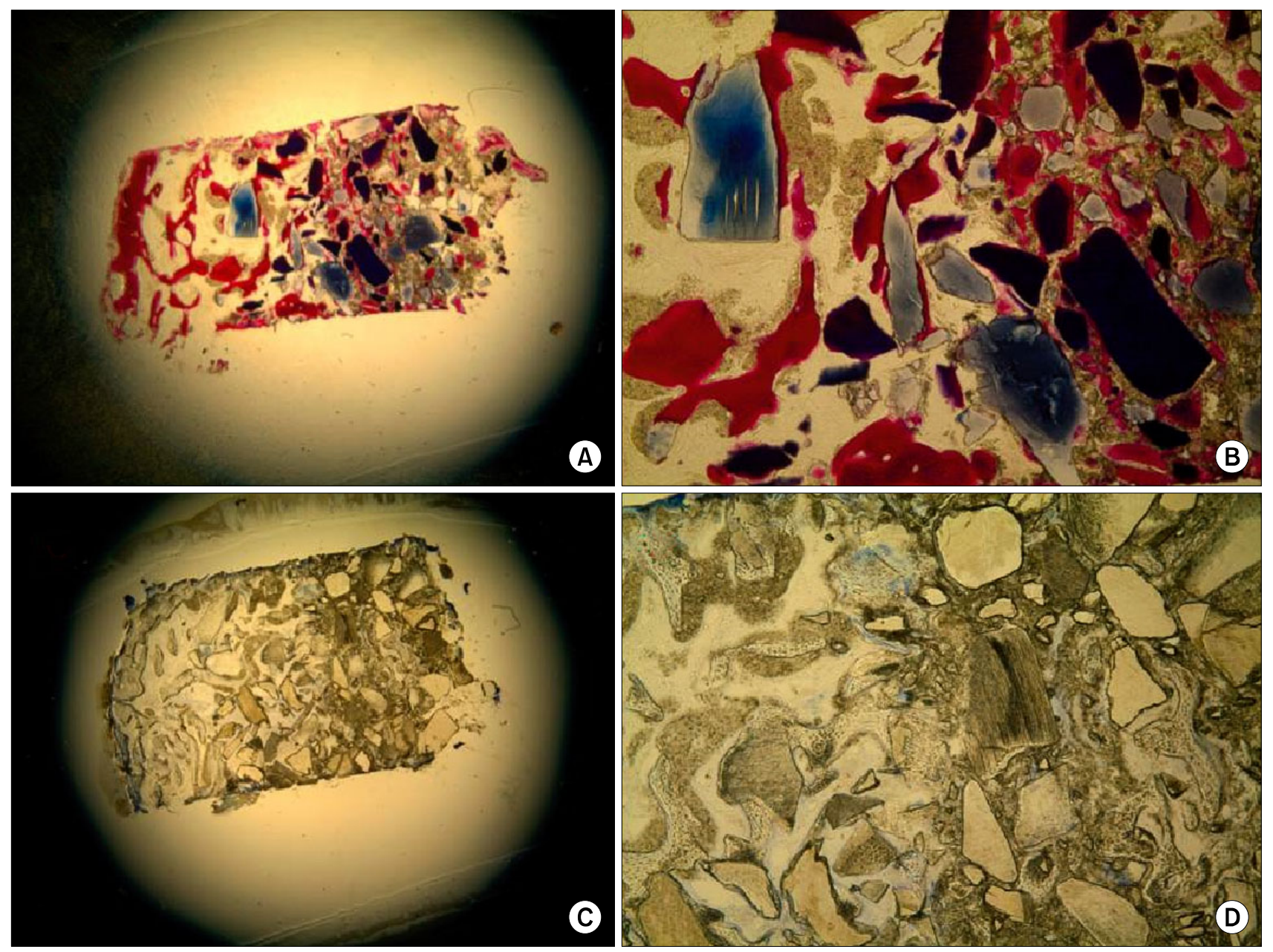

Fig. 6. Histological finding. (A) Masson trichrome (MT) staining ( $\times 12.5)$, (B) MT staining $(\times 200)$, (C) toluidine blue (TB) staining $(\times 12.5)$, (D) TB staining $(\times 200)$. Formation of new bone and rising toward the right maxillary sinus from the left alveolar bone area was observed $(A, C)$ a mixture of new bone and autogenous tooth bone particles was observed in the middle area (B, D).

\section{Discussion}

Jeong et al.[6]. conducted a retrospective study on sinus bone graft using AutoBT. A total of 100 implants in 51 patients were selected; the patients underwent maxillary sinus augmentation and implant placement using AutoBT between July 2009 and November 2010. When AutoBT was used alone or together with other graft material, the implant survival rate was 96.15\%. Based on the histomorphological examination, AutoBT showed gradual resorption and new bone formation through osteoconduction and osteoinduction. They suggested that AutoBT materials are appropriate for use in maxillary sinus augmentation Lee et al.[7] conducted a study for histomorphometric evaluation and comparison of the efficiency of various bone graft materials and autogenous tooth bone graft material used in the sinus bone graft procedure. All biopsy specimens were harvested four months after the procedure and divided into three groups according to bone graft material: Autogenous tooth bone graft material as group 1, OrthoblastII (Integra Lifescience Corp., Irvine, CA, USA)+Biocera (Osscotec, Cheonan, Korea) as group 2, and DBX (Synthes, West Chester, PA, USA)+BioOss (Geistlich Pharm AG, Wolhusen, Switzerland) as group 3. New bone formation was $52.5 \pm 10.7 \%$ in group $1,52.0 \pm 23.4 \%$ in group 2 , and $51.0 \pm 18.3 \%$ in group 3. The ratio of new bone to graft material was $81.3 \pm 10.4 \%$ in group $1,72.5 \pm 28.8 \%$ in group 2 , and $80.3 \pm 24.0 \%$ in group 3. After a four-month healing period, all groups showed favorable new bone formation around the graft material and implant. They suggested that autogenous tooth bone graft material may be used as novel bone graft material for sinus bone graft. Kim et al.[8] and 

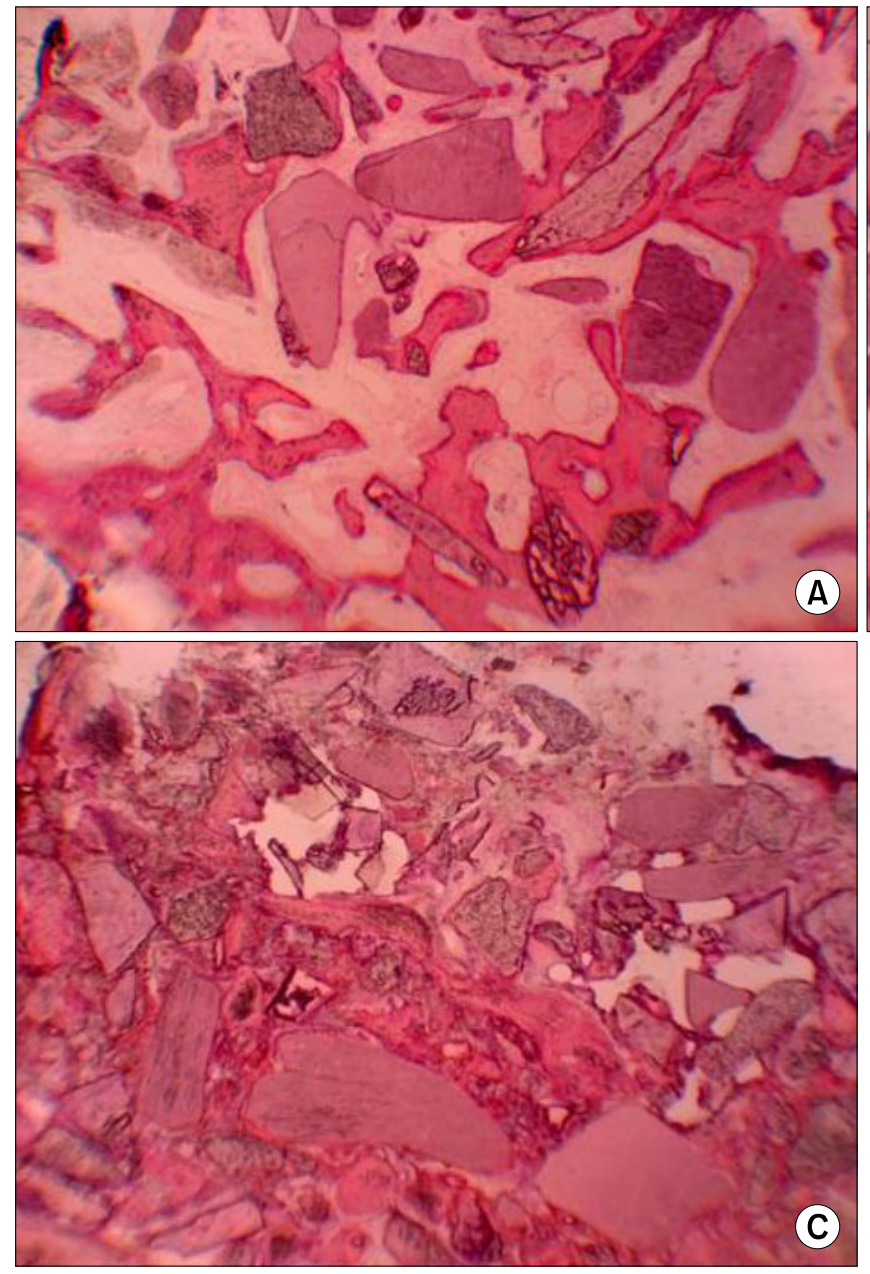

(A)

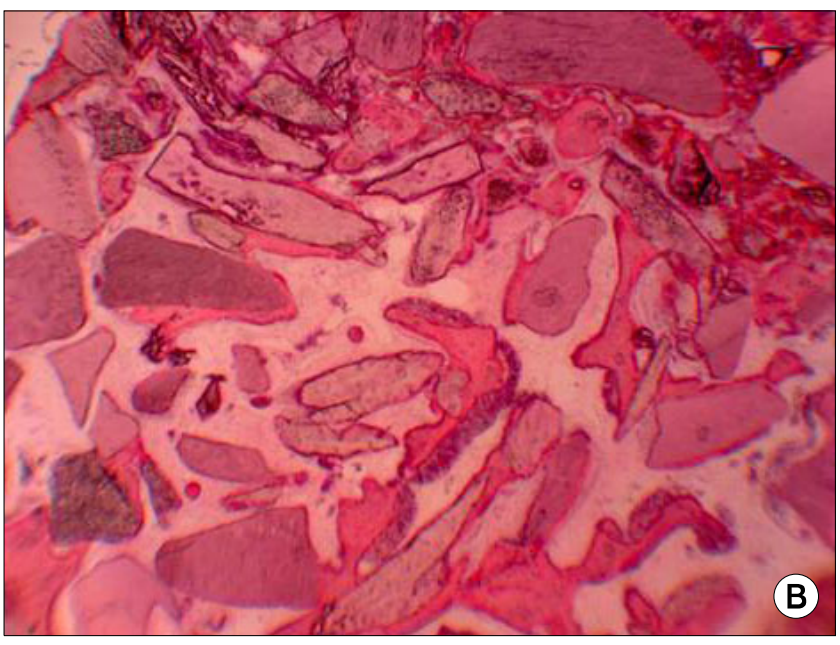

Fig. 7. Histological finding. H\&E staining $(\times 200)$. (A) Alveolar bone portion. We can see that new bone was created from the alveolar bone downward, pushing autogenous tooth bone particles upward; the border between particles of new bone and autogenous tooth bone is very dense. (B) Middle portion. New bone is shown to be adhering closely to autogenous tooth bone particles. Cell adhesion on the surface of particles is excellent, and some appear to be activated. (C) Sinus roof portion. Although the new bone bridge between autogenous tooth bone particles is observed, it is mainly filled with fibrous connective tissue.

Lee et al.[7], who performed sinus bone graft and guided bone regeneration using autogenous tooth bone from humans, took the tissue specimen two months and four months later for histomorphometric analysis. As a result, they found favorable new bone formation and suggested that autogenous tooth bone graft materials could be used in various bone grafts. Huang et al.[9] performed three-dimensional bone structure and BMD evaluations of autogenous bone graft after sinus augmentation. Iliac bone was mainly used for autogenous bone graft. After trephine core biopsy four five months after bone graft, microcomputed tomography analysis was performed. The BMD of native bone was 0.22 , and that of grafted bone was 0.12. Bone volume was reported to be $24.4 \%$ for native bone and $15.43 \%$ for grafted bone. Lundgren et al.[10] performed histomorphometric evaluation of the maxillary sinus augmentation with particulated mandible (symphysis). Trephine core biopsy specimen was collected six months and 12 months after sinus graft, and its bone volume was measured using MicroCT. The donor site bone volume was 58土19\%, and that of residual alveolar bone was $45 \pm 15 \%$. Six and twelve months later, $40 \pm 12 \%$ and $48 \pm 10 \%$, respectively, were recorded. Chackartchi et al.[11] evaluated the healing process of sinus floor augmentation using large $(1 \sim 2 \mathrm{~mm})$ or small $(0.25 \sim 1 \mathrm{~mm})$ bovine bone mineral particles. After a tissue specimen was collected $6 \sim 9$ months later, residual graft material, new bone formation, and soft tissue ratio were measured using MicroCT and histomorphology. In the MicroCT examination, the residual graft material, bone, and soft tissue were $23.12 \%, 7.99 \%$, and $68.89 \%$, respectively, for large particles, and 22.89\%, 14.64\%, and $62.47 \%$, respectively, for small particles. In the histomorphology examination, residual graft material and bone were $33.71 \%$ and $27.14 \%$, respectively, for large particles and $34.57 \%$ and $28 \%$, respectively, for small particles. According to their findings, it was difficult to distinguish 
in the MicroCT image the exact borders between the new bone and graft particles, which are in direct contact and were found to exhibit a similar pattern. Therefore, they reported that the MicroCT examination showed low proportions of bone and graft material compared with the histological exam.

In this study, as a result of analysis of the healing status nine months after maxillary sinus bone graft using Micro $\mathrm{CT}$, the total bone volume (graft material+new bone) was $76.45 \%$, and the proportion of new bone was $45.4 \%$. The $\mathrm{BMD}$ and the average $\mathrm{HU}$ of new bone were 0.26 and 1,164.69, respectively, which is D 2 bony quality. On the other hand, according to Chackartchi et al.[11], total bone volume would likely show a further increase in the histomorphometric examination. In the histological examination, the direct conglutination of new bone and AutoBT was observed, and there was no inflammatory response. In other words, growth of new bone toward the graft material from the residual alveolar bone was observed nine months after the maxillary sinus bone graft, and the graft material remained. Likewise, the examination showed that bone healing was slowest in the sinus roof area. Sinus bone graft using AutoBT showed bone healing of D 2 bony quality in the histological examination nine months later; the effect of preventing sinus re-pneumatization due to residual bone graft could be assumed.

\section{Acknowledgements}

This study was supported by a grant from the Korean Health Technology R\&D Project, Ministry of Health \& Welfare, Republic of Korea (A102065).

\section{References}

1. Hatano N, Shimizu Y, Ooya K. A clinical long-term radiographic evaluation of graft height changes after maxillary sinus floor augmentation with a 2:1 autogenous bone/xenograft mixture and simultaneous placement of dental implants. Clin Oral Implants Res 2004;15:339-45.

2. Velich N, Németh Z, Tóth C, Szabó G. Long-term results with different bone substitutes used for sinus floor elevation. J Craniofac Surg 2004;15:38-41.

3. Garg AK. Current concepts in augmentation grafting of the maxillary sinus for placement of dental implants. Dent Implantol Update 2001;12:17-22.

4. Kim YK, Kim SG, Byeon JH, et al. Development of a novel bone grafting material using autogenous teeth. Oral Surg Oral Med Oral Pathol Oral Radiol Endod 2010;109:496-503.

5. Tajima N, Ohba S, Sawase T, Asahina I. Evaluation of sinus floor augmentation with simultaneous implant placement using platelet-rich fibrin as sole grafting material. Int J Oral Maxillofac Implants 2013;28:77-83.

6. Jeong KI, Kim SG, Kim YK, Oh JS, Jeong MA, Park JJ. Clinical study of graft materials using autogenous teeth in maxillary sinus augmentation. Implant Dent 2011;20:471-5.

7. Lee JY, Kim YK, Kim SG, Lim SC. Histomorphometric study of sinus bone graft using various graft material. J Dental Rehabilitation Appl Sci 2011;27:141-7.

8. Kim SG, Kim YK, Lim SC, Kim KW, Um IW. Histomorphometric analysis of bone graft using autogenous tooth bone graft. Implantology 2011;15:134-41.

9. Huang HL, Chen MY, Hsu JT, Li YF, Chang CH, Chen KT. Three-dimensional bone structure and bone mineral density evaluations of autogenous bone graft after sinus augmentation: a microcomputed tomography analysis. Clin Oral Implants Res 2012;23:1098-103.

10. Lundgren S, Moy P, Johansson C, Nilsson H. Augmentation of the maxillary sinus floor with particulated mandible: a histologic and histomorphometric study. Int J Oral Maxillofac Implants 1996;11:760-6.

11. Chackartchi T, Iezzi G, Goldstein M, et al. Sinus floor augmentation using large $(1-2 \mathrm{~mm})$ or small $(0.25-1 \mathrm{~mm})$ bovine bone mineral particles: a prospective, intra-individual controlled clinical, micro-computerized tomography and histomorphometric study. Clin Oral Implants Res 2011;22:473-80. 